\title{
Naming unrelated words reliably predicts creativity*
}

\author{
Jay A. Olson ${ }^{1} \quad$ Johnny Nahas ${ }^{2} \quad$ Denis Chmoulevitch ${ }^{2}$ \\ Margaret E. Webb ${ }^{3}$
}

${ }^{1}$ Harvard University, ${ }^{2}$ McGill University, ${ }^{3}$ University of Melbourne

\section{Abstract}

Several theories posit that creative people are able to generate more divergent ideas. If this is correct, the simple act of naming unrelated words and then measuring the semantic distance between them could serve as an objective measure of creativity. To test this hypothesis, we asked 8,892 participants to name 10 words that are as different from each other as possible. A computational algorithm then estimated the average semantic distance between the words; related words (e.g., "cat" and "dog") have shorter distances than unrelated ones (e.g., "cat" and "thimble"). We predicted that people producing greater semantic distances would also score higher on traditional creativity measures. In Study 1, there were moderate to strong correlations between semantic distance and two other creativity measures (the Alternative Uses Task and the Bridge-the-AssociativeGap Task). In Study 2, with participants from 98 countries, semantic distances varied only slightly by demographic variables which suggests that the measure can be used without modification across diverse populations. There was also a positive correlation between semantic distance and performance on problem solving tasks known to predict creativity. Overall, semantic distance correlated at least as strongly with established creativity measures as those measures did with each other. Naming unrelated words in what we call the Divergent Association Task can thus serve as a brief, reliable, and objective measure of creativity.

*Preprint (2020-12-26). Corresponding author: J. A. Olson (jay.olson@mail.mcgill.ca). The task can be tested online at datcreativity.com. 


\section{Significance statement}

Many traditional creativity measures require time-intensive and subjective scoring procedures. Further, the scores are relative to the others in the sample and are not absolute, which makes multicultural or international assessments difficult. Our results show that a shorter and simpler task with automatic and objective scoring may be at least as reliable at measuring creativity. This finding enables creativity assessments across larger and more diverse samples with less bias. It also provides support for associative theories of creativity which suggest that creative people can generate more remote links in associative networks.

\section{Main text}

\subsection{Introduction}

Think of three words that are as different from each other as possible. Choosing these words relies on generating remote associations, which are at the core of many theories of creativity (1-5). Mednick (6), for example, proposed that most creative solutions result from the ability to find links between the most remote elements in a network of associations. Creative people, then, may more quickly and easily generate these remote associations $(3,4)$. We investigated whether the simple act of naming unrelated words could reliably measure creativity.

Creativity has two main components: convergent thinking and divergent thinking. Convergent thinking tasks measure the ability to assess several stimuli and arrive at the single best solution (6-9). In contrast, divergent thinking tasks typically involve open-ended questions that measure one's ability to generate various solutions (10-12). The most commonly used is the Alternative Uses Task $(13,14)$, in which participants generate uses for common objects such as a paper clip or a shoe. In a common method of scoring (15), raters then judge the responses based on three components:

- flexibility, the number of distinct categories of uses generated;

- originality, how rare each use is relative to the rest of the sample, which is particularly important for creativity $(16,17)$; and

- fluency, how many uses are generated in total.

Perhaps fittingly, there are diverse ways to score tests of divergent thinking (10,18-23). Many of the manual scoring methods, however, have several drawbacks. The scoring is laborious and time-intensive (10), and multiple judges are required to assess reliability which adds to the effort (24). Further, the scoring is sample-dependent $(22,24)$; originality is scored in a relative and not an absolute way. Thus, a participant's responses will be more or less rare (and more or less original) depending on the other responses in the sample. Finally, the scoring does not account for cultural differences: uses of objects vary 
in commonality across cultures and at different times. The use of a paper clip to change the SIM card in a smartphone, for example, is now one of the most common responses, though it was rare a decade ago. Ratings of originality will thus vary by country and year. This issue makes it difficult to accurately judge responses from multicultural or international samples, or to assess how creativity changes over time.

To address these limitations, recent efforts have moved towards using computational algorithms to score responses $(10,16,25,26)$. Computational methods may also improve the theoretical grounding of the measures, as the assumptions required to score the responses must be made explicit in the program code $(10,12)$. Researchers have successfully scored the Alternative Uses Task using semantic models, achieving scores similar to human ratings $(10,16,25,26)$.

The Alternative Uses Task, however, may not be best suited for computational scoring. It requires analysing complex multi-word answers, usually by computing the semantic distance between each of the words provided in the response, independent of their order. Common methods also remove stop words (e.g., the, which, of) in order to increase the consistency of the scoring $(26,27)$. These common practices can obscure meaning, which is a well-known issue in the field of artificial intelligence. Word meaning depends on context and order, as demonstrated by the sentence: "Time flies like an arrow; fruit flies like a banana" (28). In the Alternative Uses Task, using a pen to "record a break" is less

original than using one to "break a record". Originality can accordingly be more than the sum of its parts (29). Additionally, the number of words used to describe a concept can also influence semantic distance (27). Using a magazine to "do a collage" versus to "cut out pieces to glue on a collage" will result in different scores. Thus, a task that uses single-word responses and instructs participants to consider all meanings of the words may reduce these problems and result in more reliable scoring.

Accordingly, we aimed to develop a measure of divergent thinking that focuses on remote associations, is more compatible with existing theories, and is optimised for computational scoring. We wanted a task that required single-word responses, making it easier to compute semantic distance with less ambiguity. More practically, our goal was to develop a task that is brief, easy to implement, and offers objective, automatic, and absolute scoring.

\subsubsection{The Divergent Association Task}

Our proposed measure, the Divergent Association Task (DAT), asks participants to generate 10 words that are as different from each other as possible, in all meanings and uses of the words. We then compute the average semantic distance between these words using an algorithm called GloVe (30). Words that are used in similar contexts have smaller distances between them. The words cat and $d o g$, for example, would be close to each other since they are often used in similar contexts. The words day and night, though opposites, would also be close to each other for the same reason. Importantly, words closer to each 
other are usually more semantically similar.

We trained the GloVe model with the Common Crawl corpus, which contains the text of billions of web pages. In particular, we used a freely available pre-trained model from 2016 (30), which contains a vocabulary of over 2 million words.

Using this model, we computed the average cosine distance between each unique pair of words and multiplied it by a constant of 100 :

$$
\text { DAT score }=100 \sum_{i j} \frac{\left|W_{i}-W_{j}\right|}{N}
$$

The minimum DAT score (0) occurs when there is no distance between the words, that is, when all the words are the same. The theoretical maximum score (200) would occur when the words are as different from each other as possible. In practice, scores commonly range from approximately 65 to 90 and almost never exceed 100. As discussed later, scores under 50 are discarded. In this way, the score can be intuitively thought of as a percentage on a test: under 50 is a fail, the average is between 75 and 80 , and 95 is a very high score. Figure 1 shows example words and their corresponding scores.
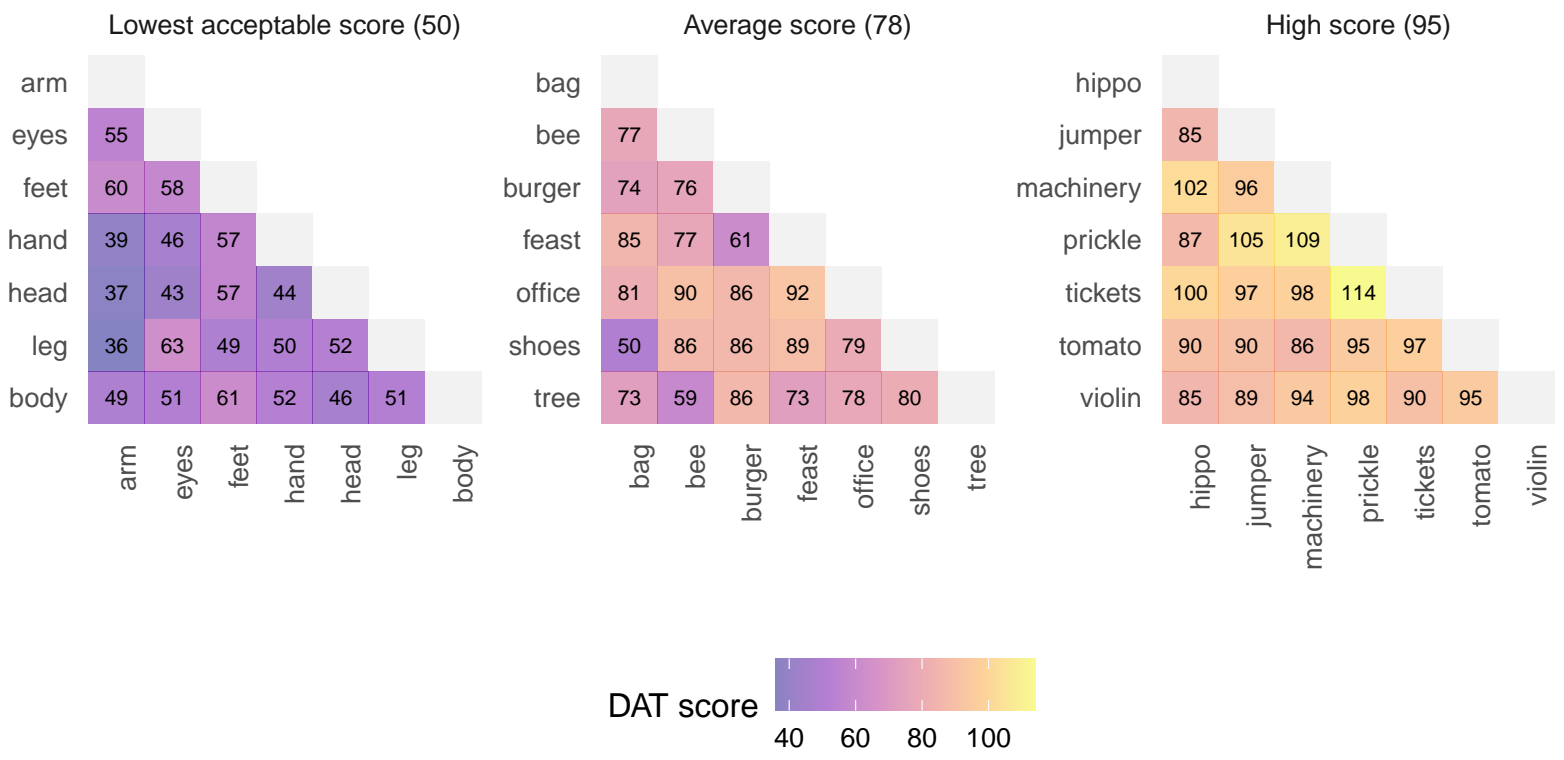

Figure 1: Examples of participant responses and their corresponding DAT scores. The score is the transformed average of the semantic distances between each pair of words.

This operationalisation of divergent thinking is grounded in associative theories of creativity $(3,6,10)$. Higher scores would demonstrate a greater ability to draw upon more remote associations, indicating greater creativity. In Study 1, we tested this hypothesis by comparing the DAT with two other measures of creativity: the Alternative Uses Task (14) and the Bridge-the-Associative-Gap Task (31). In Study 2, we tested how these scores 
vary by demographics and whether they correlate with related problem solving measures $(8,32)$. These studies assessed whether semantic distance can be a reliable indicator of creativity.

\subsection{Results}

\subsubsection{Number of words}

The DAT asks participants to name 10 unrelated words, but we only required a subset of these to provide a buffer for mistakes. This way, if participants mistyped a few words or chose some that were not in the database, we could still compute an overall score. In Study 1A, all participants ended up providing at least seven valid words (Figure 2A), so we used this number of words in all of the samples.
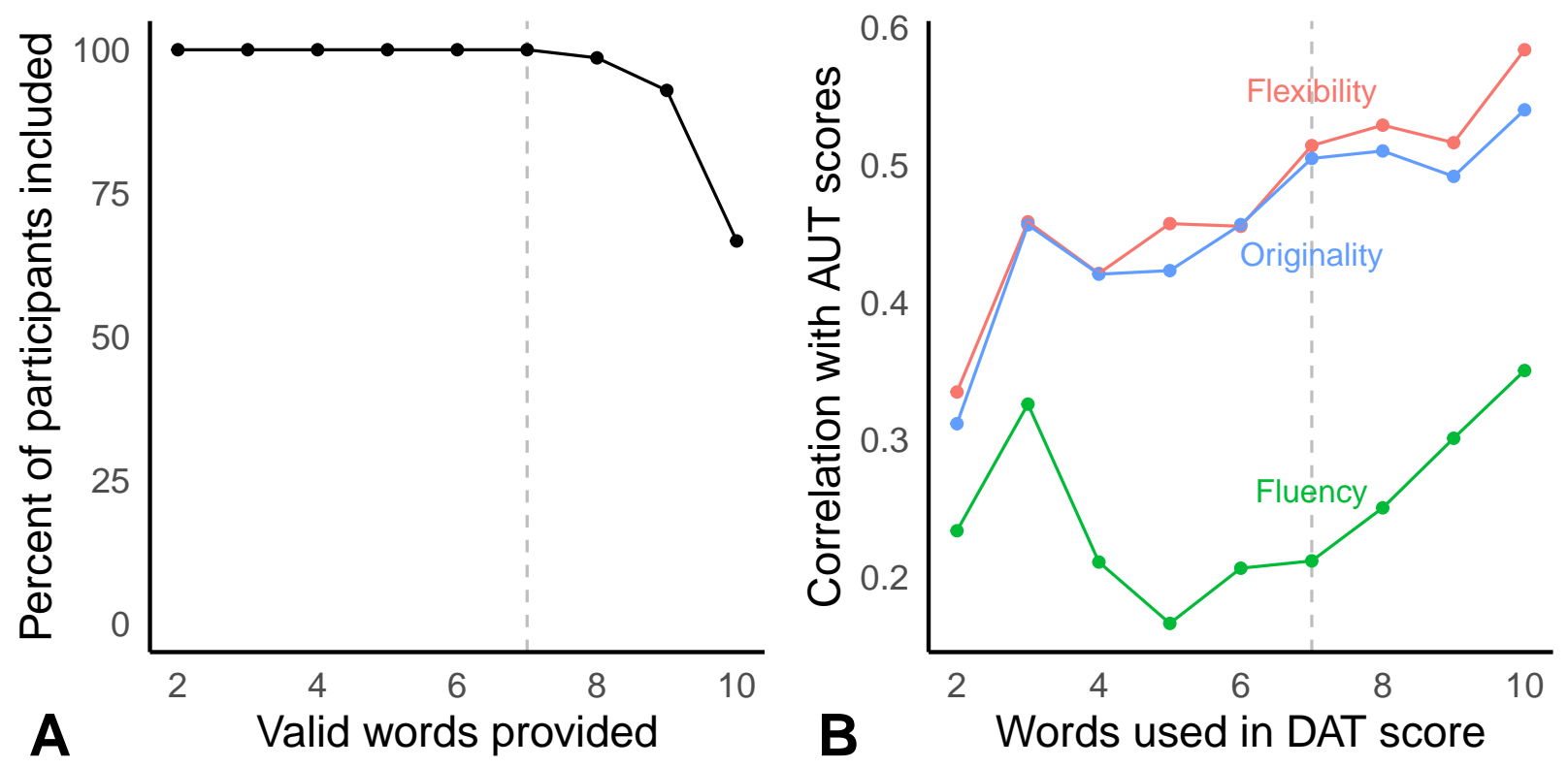

Figure 2: (A) Percent of participants included when requiring different numbers of words in the DAT score and (B) corresponding correlations with the Alternative Uses Task scores. Using 7 out of 10 words balanced high correlations with a high inclusion rate.

Using these first seven words, the average DAT score for Study 1A was $78.38(S D=6.35)$ and the distribution was roughly normal as in the subsequent samples (Table S1). Most participants finished the DAT in a minute and a half, with a median response time of $88.21 \mathrm{~s}(S D=66.46)$. The task is thus shorter than many traditional creativity measures.

\subsubsection{Correlations with other creativity measures}

In Study 1A, using a subset of the data in which participants chose the words arbitrarily (i.e., without following any particular strategy), the DAT correlated well with the Alternative Uses Task (Figure 2B). In particular, the DAT correlated with flexibility 
$(r(55)=.51[.29, .68], p<.001)$ and originality $(r(55)=.50[.28, .68], p<.001)$, but we did not see the same correlation with fluency $(r(55)=.21[-.05, .45], p=.057)$. Using the full dataset without removing any participants, we saw positive correlations across all three ratings (flexibility: $r=.34[.18, .48]$; originality: $r=.32[.16, .46]$; fluency: $r=.22[.06, .37]$ ).

Participants also completed the Bridge-the-Associative-Gap Task, a test of convergent thinking in which participants see two words (e.g., giraffe and scarf) and need to find a third that relates to both (e.g., neck). Raters then judged the appropriateness of the provided words. We saw a positive correlation between the DAT and appropriateness among participants choosing words without following a particular strategy $(r(54)=$ $.34[.08, .55], p=.006)$ as well as in the full dataset $(r(136)=.22[.06, .38], p=.004)$.

Study $1 \mathrm{~B}$ attempted to replicate these findings in another full dataset. We again saw positive correlations with the Alternative Uses Task (flexibility: $r(222)=.36[.24, .47]$, $p<.001$, originality: $r(222)=.33[.21, .44], p<.001$, fluency: $r(222)=.30[.17, .41]$, $p<.001)$ and appropriateness ratings in the Bridge-the-Associative-Gap Task $(r(203)=$ $.23[.10, .36], p<.001)$.

To assess test-retest reliability, in Study 1C participants completed the DAT during lab visits two weeks apart. Test-retest reliability was high $(r(48)=.73[.57, .84], p<.001)$; this reliability resembled that of completing the same Alternative Uses Task items one month later, as scored by raters ( $r=.61$ to .70$)$ or an algorithm ( $r=.49$ to .80$)$ (33).

In our pre-registered Study 2, a confirmatory test showed a positive correlation between the DAT and manually scored originality on a one-trial Alternative Uses Task $(r(353)=$ $.13[.03, .23], p=.006)$. The magnitude was lower than in Study 1 likely because the shortened version of the task had less precision.

\subsubsection{Demographics}

Confirmatory tests also showed that the DAT scores differed by age and gender but not the country in which participants lived or whether they spoke multiple languages (Table S2). Scores were slightly higher in women and peaked in their 20s (Figure 3). Still, all of these factors combined explained under $1 \%$ of the total variation in the model, suggesting that the DAT varies little by these demographic measures.

\subsubsection{Problem solving}

Participants also completed various problem solving items known to predict creativity (8, 32). On average, participants answered $2.11(S D=1.03)$ out of the 4 questions correctly. An exploratory test showed that those who correctly completed more problems had higher DAT scores $(r(348)=.16[.05, .26], p=.003)$. 


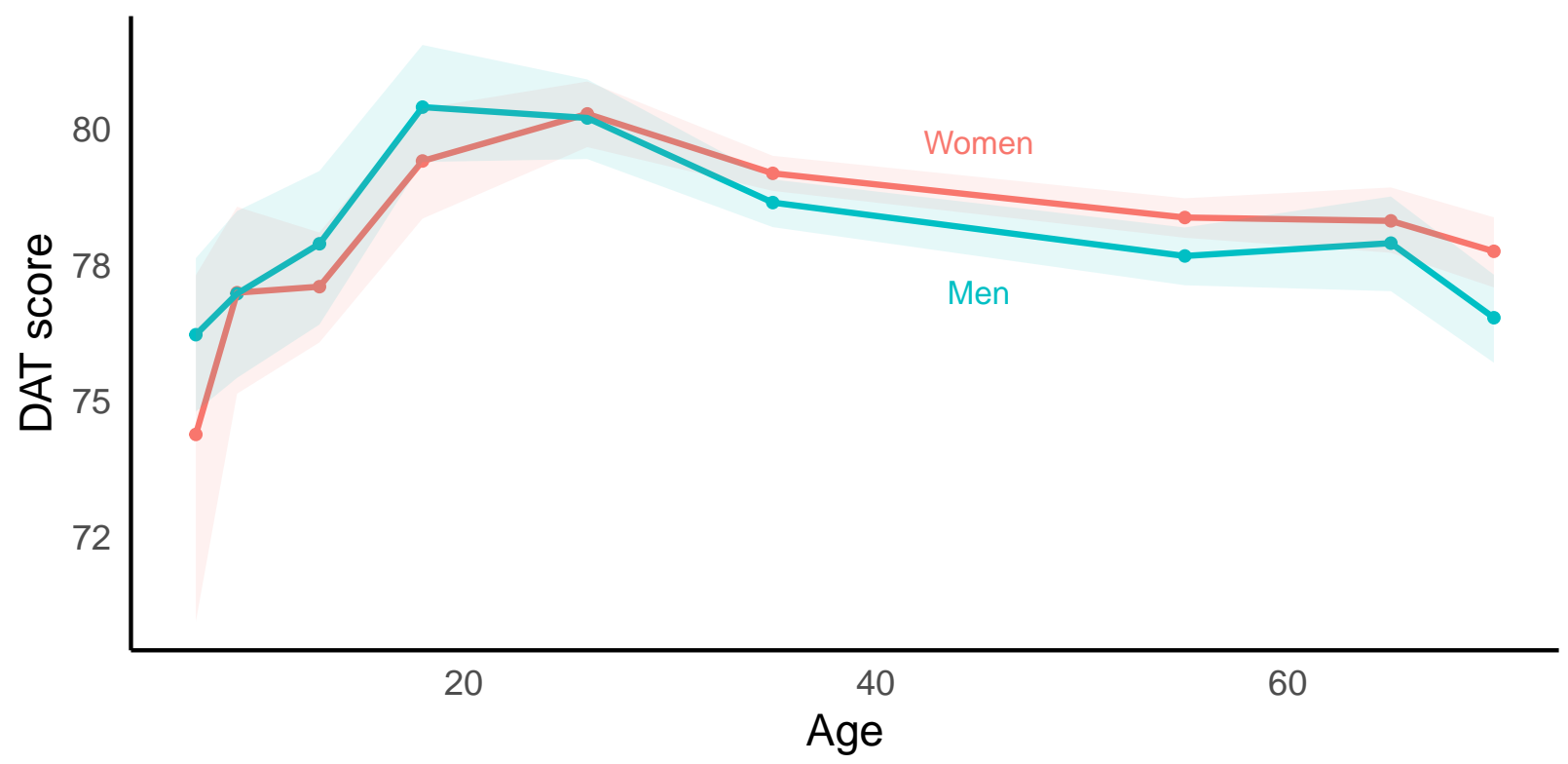

Figure 3: Divergent Association Task scores by age and gender. Scores peaked in young adulthood and women showed slightly higher overall scores than men. Dots show means and bands show $95 \%$ confidence intervals. Age was approximated by the minimum value of each bin.

\subsubsection{Enjoyment}

After each measure of Study 2, participants reported how much they enjoyed it on a 0 to 5 scale. Participants enjoyed the DAT the most, with an average rating of 3.56 [3.52, 3.59] compared to the Alternative Uses Task items $(M=2.65[2.62,2.68])$ or any of the various problem solving items $(M=2.74[2.62,2.84]$; Figure $S 1)$.

\subsubsection{Comparison with other correlations}

Across Studies 1 and 2, we saw positive correlations between the DAT and the other creativity measures. These sample correlations were generally at least as strong as the correlations among the other established measures (Figure 4).

\subsection{Discussion}

We present a surprising finding: asking participants to name 10 unrelated words can be a reliable measure of creativity. This novel Divergent Association Task is situated in associative processing theories of creativity, which assume that creative people can draw on distant nodes in associative networks to generate more semantically remote concepts. We compared performance on this task with established creativity measures the Alternative Uses Task and the Bridge-the-Associative-Gap Task — as well as related measures of problem solving. In every sample, the correlations between the DAT and 


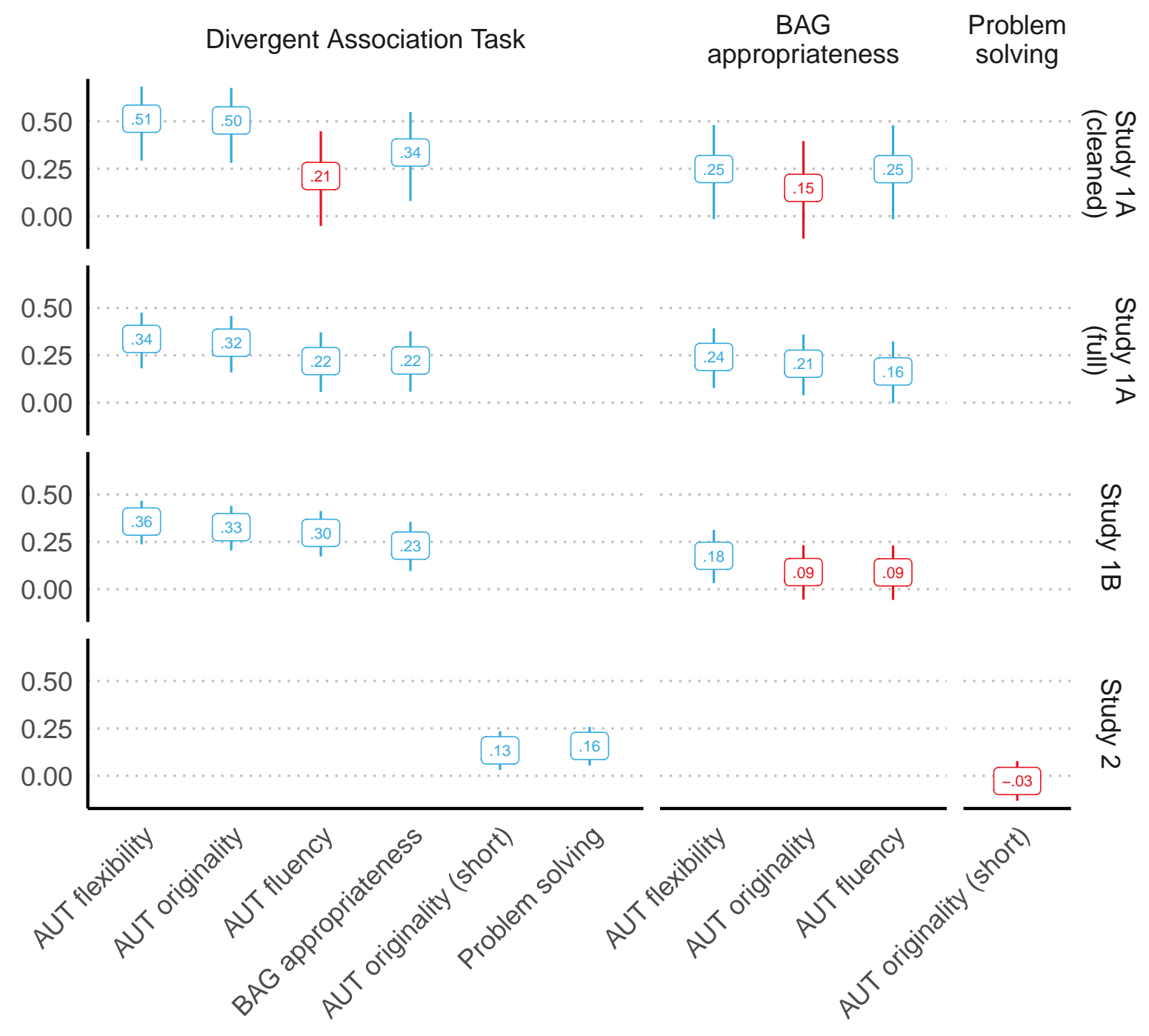

Directional hypothesis decision: a positive correlation a zero correlation

Figure 4: Between-task correlations (columns) across studies (rows). The DAT generally showed the strongest correlations (first column) compared to the other measures. Colour shows whether correlations were greater than zero in a one-tailed test. 
these measures were at least as high as the correlations among the other established measures themselves, demonstrating strong convergent validity. The highest correlations were between the DAT and manually scored originality and flexibility on the Alternative Uses Task (Studies 1A-1B). Test-retest reliability was also high over a span of two weeks (Study 1C). Overall, the evidence supports semantic distance as a reliable measure of creativity.

Performance on the DAT varied little by demographic measures (Study 2). Other studies have shown mixed results relating demographics and creativity. For example, studies are inconclusive about the impact of gender differences $(34,35)$. Abraham (36) reports highly mixed findings, with approximately half of studies reporting no differences in creativity by gender, and the other half reporting mixed results, with possibly higher scores in women. There are also mixed findings regarding age; some studies indicate that performance on creativity tasks increases as domain-specific knowledge and vocabulary increase (37), while others find the opposite (38). Generally, however, studies find that creativity declines in late adulthood (35). Overall, the low variation by demographic variables suggests that the DAT can be used across different ages, genders, and countries without modification.

\subsubsection{Strengths}

The Divergent Association Task resolves a number of limitations in the current creativity literature. Compared to manually scored tasks, the DAT scoring is automatic and objective, allowing researchers to collect large samples with little effort and no rater bias. Further, the scoring is absolute and not sample-dependent, making it conducive to comparing participants across times and countries. Compared to other creativity measures using computational scoring, the DAT draws more directly on theories of creativity such as those related to associative processing and semantic networks $(10,39)$. This close link provides greater face validity and makes the task better suited for computational scoring than adapting existing measures. In addition, the DAT may reduce some biases seen in other tests of divergent thinking. Experiential bias occurs when one's past experience influences the diversity of the responses (40). When listing uses of a brick in the Alternative Uses Task, for example, a brick layer may provide different responses than a lawyer, influencing their scores within the sample and leading to more uncontrollable variation. Similarly, different words in the Alternative Uses Task can prompt different responses with varying reliability between computational scoring and manually scored responses (26). The DAT avoids these issues by giving an open-ended prompt and using an international database. Relatedly, fluency contamination occurs when high fluency scores can artificially inflate originality scores; the more responses that participants list, the more likely some of them will be unique (21). The DAT avoids this issue by requiring all participants to generate the same number of responses.

Finally, the task is short - most participants completed it in under $90 \mathrm{~s}$ - and rated it as more enjoyable than several other creativity tasks. The task can also provide instant 
feedback of the participant's score, allowing it to be used in contexts such as workshops or demonstrations. Enjoyment, brevity, and feedback are especially important for online tasks with reduced or no compensation, in which intrinsic motivation plays a central role in the desire to participate.

\subsubsection{Limitations}

Although the DAT improves upon a number of issues in the creativity literature, it has its own limitations. For instance, the task measures originality with better face validity than it does appropriateness, though both are important components of creativity (41). Appropriateness is easier to judge in other tasks, such as when naming uses for an object or finding the link between words, but it has less relevance when naming unrelated words in the DAT. Still, the DAT scores correlated with assessments of appropriateness on the Bridge-the-Associative-Gap Task. Another limitation is that participants may be able to artificially modulate their scores using different strategies of generating words. Intentionally choosing rare words, looking around the room for inspiration, or following word-based strategies (e.g., choosing rhyming words) can all influence the overall score. The fairly short time limit of four minutes may reduce the likelihood that participants will consider and implement these various strategies.

\subsubsection{Future research}

Like all computational scoring methods, the DAT depends on the training model and corpus used. We chose the GloVe algorithm and the Common Crawl corpus; this combination correlates best with human judgements on the Alternative Uses Task (26). For simplicity, we chose a pre-trained model that is freely available and widely used. With some effort, researchers could train models using corpora in different languages or countries at different times. As particular word associations become more or less related, the updated models would automatically account for these changes. Prior to the COVID-19 pandemic, for example, the words social and distancing would co-occur more rarely and would thus be considered more original. In theory, researchers could generate a specific model using content from the same country and month as the sample. This would allow the DAT scores to reflect the fluctuations in the cultural lexicon, as world events and popular media shape word usage. Still, for simplicity and ease of comparison, we recommend that researchers use the corpus tested here unless there is a reason otherwise.

Future research could also use the DAT in experimental contexts requiring simple and short tasks. Since the DAT requires brief responses, it may be suited for neuro-imaging contexts in which movement must be minimised. Its simplicity may also make it suitable for completion in altered states of consciousness conducive to divergent thinking, such as pre-sleep states or when using psychedelic drugs $(42,43)$. Since the task involves an open-ended prompt, researchers could also assess whether the responses reflect the interests, experiences, or biases of the participants. Finally, future research could also 
explore other predictors of DAT scores, such as education level or intelligence, which both generally correlate with creativity $(1,44)$.

In sum, we demonstrate that naming unrelated words can be a reliable measure of creativity. We hope this finding provides researchers with an easier method of collecting and scoring creativity data with larger and more diverse samples.

\section{Materials and methods}

\subsection{Study 1}

We validated the DAT against two established measures of creativity: the Alternative Uses Task and the Bridge-the-Associative-Gap Task. Study 1 contains three samples of participants who completed a demographics questionnaire and then several creativity tasks on a computer. The protocol was approved by the University of Melbourne Human Research Ethics Committee (1646146.4).

\subsubsection{Participants}

We recruited undergraduates from psychology courses in Melbourne, Australia (Studies 1A and 1B) and through social media advertisements in Montréal, Canada (Study 1C). In each sample of Study 1, most participants were women and between 18 and 20 years old (Table 1).

Table 1: Participant demographics across samples. In Study 2, ages are approximate since they were reported in bins.

\begin{tabular}{lrrrrr}
\hline Study & $1 \mathrm{~A}$ & $1 \mathrm{~B}$ & $1 \mathrm{C}$ & 2 & Total \\
Country & Australia & Australia & Canada & 98 countries & 98 countries \\
$N$ & 141 & 284 & 50 & 8551 & 8892 \\
$\%$ Female & $82 \%$ & $68 \%$ & $76 \%$ & $59 \%$ & $59 \%$ \\
Age $M$ & 20.12 & 19.22 & 20.84 & 43.50 & 42.57 \\
Age $S D$ & 4.05 & 2.18 & 2.68 & 17.66 & 17.93 \\
Age range & $18-47$ & $16-47$ & $18-33$ & $6-70$ & $6-70$ \\
\hline
\end{tabular}

\subsubsection{Materials}

4.1.2.1 Alternative Uses Task In the Alternative Uses Task, participants were presented with common objects in a randomised order: a brick, paper clip, newspaper, ice tray, and rubber band (14). The participants were instructed: "This is a test of creativity. Please list as many (and varied) uses as you can." Participants had two minutes to respond to each item in a text box; with five items, the task took up to ten minutes.

Two independent raters later scored the responses using a uniqueness method adapted from deYoung and colleagues (15). Originality scores depended on the frequency of the 
response within the sample. Participants received 0 points for responses given by over $10 \%$ of the sample (e.g., using a rubber band to tie up hair), 1 point for responses given by 3 to $10 \%$ (e.g., as a string), 2 points for those given by 1 to 3\% (e.g., as a tourniquet), and 3 points for responses given by under 1\% (e.g., as dental floss). Flexibility scores depended on the number of different categories of uses mentioned. Using a rubber band as an eraser or to grip bottles would represent two distinct categories; using it to tie plastic bags or to group wires would represent one category (tying things together). Fluency was simply the number of distinct responses given in two minutes. Across the samples, the two raters showed high inter-rater reliability (flexibility: $r=.94$ to .97 , originality: $r=.64$ to .89 , fluency: $r=.99$ to 1.00 ), so we averaged their scores.

4.1.2.2 Bridge-the-Associative-Gap Task In the Bridge-the-Associative-Gap Task, a test of convergent thinking, participants were presented with pairs of words that were either related or unrelated to each other. Participants were asked to write a third word that is semantically related to both of the words. For example, if presented with giraffe and scarf, participants could write neck as the third word. The participants were given 30 seconds to respond to each item. In Study 1A, we randomly selected 20 of each type of pairs (related or unrelated) from the original set (31). Study 1B used the entire set.

Two judges then assessed the appropriateness of each response from 1 to 5 based on whether it related to both words in the pair. For example, a response of neck would be judged as appropriate (5) given giraffe and scarf, but a response of cheese would not be (1). The judges generally agreed on their ratings ( $r=.67$ to .78$)$, so we averaged their scores.

4.1.2.3 Divergent Association Task As outlined above, participants were asked to generate 10 unrelated words (see Section 6.1 for a pencil-and-paper version of the task). The task had the following additional instructions:

1. Use only single words. We used this rule because computational methods can score single words with less ambiguity than phrases. Words such as "cul de sac" were accepted and automatically hyphenated.

2. Use only nouns (e.g., things, objects, concepts). This rule keeps the class of words similar, since the distance between words varies based on their part of speech, such as whether they are nouns or adjectives.

3. Avoid proper nouns (i.e., no specific people or places).

4. Avoid specialised vocabulary (e.g., no technical terms). This rule and the previous one prevent participants from using words that are too specific, which is one strategy to artificially inflate one's score. To enforce these rules, only lowercase words from a common dictionary (45) were used in the calculation.

5. Think of the words on your own (e.g., do not just look at objects in your surroundings). During pilot testing, many participants would look around their environment for 
inspiration when naming the words. This strategy resulted in lower scores, since common objects on one's desk are often semantically similar.

6. You will have 4 minutes to complete this task. In our initial testing (46), this amount of time was sufficient to complete the task without much time pressure.

After the task, participants were asked what strategy they used, if any, to choose the words (e.g., looking around the room at objects). In Study 1A, two raters coded the responses based on whether the 141 participants (1) appeared to correctly follow the instructions and (2) whether they reported implementing a strategy such as simply naming the objects around them. Disagreements were solved by discussion and raters were liberal in their exclusions. Overall, 57 participants appeared to follow the instructions and not use a strategy; we used this subset for our analyses. The lowest score in this subsample was 57.13.

In Studies $1 B$ and $1 C$, instead of this manual exclusion procedure, we used an automated method. In particular, we removed scores below 50, under which participants almost certainly chose words that had clear relations between them (see the first panel in Figure 1). This excluded at most $0.35 \%$ of the sample.

\subsubsection{Analysis}

To test the relationship between the creativity measures, we checked for non-linearity then did one-tailed tests of linear correlation, with an $\alpha$ of .05 and no family-wise Type I error correction. All assumptions were reasonable for the tests. In Studies 1A and 1B, we aimed to run at least 90 students per sample, which gave $80 \%$ statistical power to detect medium correlations of $r=.3$.

\subsection{Study 2}

We also tested how DAT scores vary by age, gender, country, and languages spoken. We recruited a larger and more diverse sample as part of a broader study on experiences reported during creativity tasks.

\subsubsection{Participants}

Participants were recruited through television advertisements and social media as part of a campaign by the Australian Broadcasting Corporation. In total, 8551 participants completed the study from 98 countries. Most of the participants were from Australia $(n=4759)$ or the United Kingdom $(n=612)$. Participants reported ages in bins ranging from under 7 years old $(n=6)$ to 70 or over $(n=961)$, with most falling in the 35 to 54 age range $(n=2829)$ making the sample older than the students in Study 1 . Again, the majority of the sample was female (59\%; Table 1$)$. 


\subsubsection{Materials}

4.2.2.1 Alternative Uses Task Due to time constraints, we used a shorter version of the task in which participants were asked to generate a single "new and imaginative use" for two common household objects. These objects were randomly selected from a brick, rubber band, shoe, paper clip, cup, or ice tray. Given that participants generated a single use, flexibility and fluency could not be evaluated, so we focused on originality. Two raters judged originality from 1 to 5 in a random subsample including 389 participants. We chose a subsample that included those responding to the same objects as much as possible. As in the previous samples, the judges generally agreed on their ratings $(r=.66)$, so we averaged their scores.

4.2.2.2 Divergent Association Task We used the standard version of this task, as in Study 1, excluding people with scores under 50 .

4.2.2.3 Problem solving Participants then completed a series of creativity-related problems. The Compound Remote Associates Test is a convergent thinking task commonly used for assessing insight problem solving and creativity (8). Participants saw three cue words and tried to find a fourth word that formed a compound word with the cues. For example, given the words cube, skate, and cream, participants would suggest the word ice. Participants were presented with two trials of the task, randomly selected from a larger set (8).

Participants also completed one insight and one non-insight problem taken from a larger set (32). Two raters independently judged the accuracy of the answers to the questions. A third rater resolved answers judged as ambiguous. Inter-rater reliability between the two raters was high $(r=.87)$; we considered answers as correct only when both raters scored them as such. After each trial, participants reported their feelings during the problem solving (e.g., pleasure) from 0 (nothing) to 5 (strong), as part of the larger study.

4.2.2.4 Demographic information We collected brief demographic information aimed at maintaining anonymity: age (in bins), gender, country (dichotomous Australia or not), and whether the participant was multilingual (i.e., whether they spoke a language other than English).

\subsubsection{Procedure}

On a website, participants were informed that the purpose of the study was to investigate their experiences with several creativity and problem solving tasks. Participants then completed the shortened Alternative Uses Task, the DAT, and then the following questions in a random order: one insight problem, one non-insight problem, and two Compound Remote Associates Test items. For each measure, the items were taken from a larger set to 
reduce contamination (e.g., if several people completed the task in the same room). After completing all of the tasks, participants provided demographic information.

\subsubsection{Analysis}

All aspects of the study were pre-registered online (see https://osf.io/bfke8). To test the relationship between originality and the DAT scores, we checked for non-linearity then did a one-tailed test of linear correlation with an $\alpha$ of .05 .

To assess how scores varied across demographics, we used ANOVA to test for main effects of (1) age (estimated by the minimum value of each age bin), (2) gender (female or male), (3) country (Australia or not), and (4) multilingualism, as well as interaction effects of (5) gender $\times$ age, and (6) country $\times$ multilingualism. Using the Bonferroni correction, a family-wise $\alpha$ of .10 gave a per-test $\alpha$ of .0167. Given our large expected sample size, we maintained high statistical power despite the low Type I error rates.

\section{Acknowledgements}

We would like to thank Elias Stengel-Eskin for help with the conceptualisation of the task and the Australian Broadcasting Corporation for assistance with recruiting. We also thank Victoria De Braga, Ellen Langer, and Claire Suisman for discussion and feedback.

JO acknowledges funding from le Fonds de recherche du Québec - Santé (FRQS). Study 1C was supported by the Canada First Research Excellence Fund, awarded to the Healthy Brains for Healthy Lives initiative at McGill University (\#3c-KM-10).

\section{References}

1. M. Benedeket al., How semantic memory structure and intelligence contribute to creative thought: A network science approach. Thinking \& Reasoning 23, 158-183 (2017).

2. N. Gupta, Y. Jang, S. C. Mednick, D. E. Huber, The road not taken. Psychological Science 23, 288-294 (2012).

3. Y. N. Kenett, D. Anaki, M. Faust, Investigating the structure of semantic networks in low and high creative persons. Frontiers in human neuroscience 8, 407 (2014).

4. E. Rossmann, A. Fink, Do creative people use shorter associative pathways? Personality and Individual Differences 49, 891-895 (2010).

5. M. A. Schilling, A "small-world" network model of cognitive insight. Creativity Research Journal 17, 131-154 (2005).

6. S. Mednick, The associative basis of the creative process. Psychological Review 69, 220-232 (1962). 
7. M. Becker, G. Wiedemann, S. Kühn, Quantifying insightful problem solving: A modified compound remote associates paradigm using lexical priming to parametrically modulate different sources of task difficulty. Psychological Research 84, 528-545 (2018).

8. E. M. Bowden, M. Jung-Beeman, Normative data for 144 compound remote associate problems. Behavior Research Methods, Instruments, \& Computers 35, 634-639 (2003).

9. C.-L. Wu, H.-C. Chen, Normative data for Chinese compound remote associate problems. Behavior Research Methods 49, 2163-2172 (2017).

10. S. Acar, M. A. Runco, Assessing associative distance among ideas elicited by tests of divergent thinking. Creativity Research Journal 26, 229-238 (2014).

11. M. Benedek, T. Könen, A. C. Neubauer, Associative abilities underlying creativity. Psychology of Aesthetics, Creativity, and the Arts 6, 273-281 (2012).

12. R. W. Hass, Semantic search during divergent thinking. Cognition 166, 344-357 (2017).

13. J. P. Guilford, Creativity. American Psychologist 5, 444-454 (1950).

14. M. A. Wallach, N. Kogan, A new look at the creativity-intelligence distinction. Journal of Personality 33, 348-369 (1965).

15. C. G. DeYoung, J. L. Flanders, J. B. Peterson, Cognitive abilities involved in insight problem solving: An individual differences model. Creativity Research Journal 20, 278-290 (2008).

16. K. Beketayev, M. A. Runco, Scoring divergent thinking tests by computer with a semantics-based algorithm. Europe's Journal of Psychology 12, 210-220 (2016).

17. M. A. Runco, G. J. Jaeger, The standard definition of creativity. Creativity Research Journal 24, 92-96 (2012).

18. S. Acar, M. A. Runco, Divergent thinking: New methods, recent research, and extended theory. Psychology of Aesthetics, Creativity, and the Arts 13, 153-158 (2019).

19. M. Benedek, C. Mühlmann, E. Jauk, A. C. Neubauer, Assessment of divergent thinking by means of the subjective top-scoring method: Effects of the number of top-ideas and time-on-task on reliability and validity. Psychology of Aesthetics, Creativity, and the Arts 7, 341-349 (2013).

20. R. W. Hass, M. Rivera, P. J. Silvia, On the dependability and feasibility of layperson ratings of divergent thinking. Frontiers in Psychology 9 (2018).

21. J. A. Plucker, M. Qian, S. L. Schmalensee, Is what you see what you really get? Comparison of scoring techniques in the assessment of real-world divergent thinking. Creativity Research Journal 26, 135-143 (2014).

22. R. Reiter-Palmon, B. Forthmann, B. Barbot, Scoring divergent thinking tests: A review and systematic framework. Psychology of Aesthetics, Creativity, and the Arts 13, 144-152 
(2019).

23. P. J. Silvia, Subjective scoring of divergent thinking: Examining the reliability of unusual uses, instances, and consequences tasks. Thinking Skills and Creativity 6, 24-30 (2011).

24. P. J. Silviaet al., Assessing creativity with divergent thinking tasks: Exploring the reliability and validity of new subjective scoring methods. Psychology of Aesthetics, Creativity, and the Arts 2, 68-85 (2008).

25. R. E. Beaty, D. R. Johnson, Automating creativity assessment with SemDis: An open platform for computing semantic distance. PsyArXiv (2020).

26. D. Dumas, P. Organisciak, M. Doherty, Measuring divergent thinking originality with human raters and text-mining models: A psychometric comparison of methods. Psychology of Aesthetics, Creativity, and the Arts (2020) https:/doi.org/10.1037/aca0000319.

27. B. Forthmann, O. Oyebade, A. Ojo, F. Günther, H. Holling, Application of latent semantic analysis to divergent thinking is biased by elaboration. The Journal of Creative Behavior 53, 559-575 (2018).

28. F. J. Crosson, Human and artificial intelligence (Appleton-Century-Crofts, 1970).

29. T. K. Landauer, D. Laham, B. Rehder, M. E. Schreiner, How well can passage meaning be derived without using word order? A comparison of latent semantic analysis and humans in Proceedings of the 19th Annual Meeting of the Cognitive Science Society, (1997), pp. 412-417.

30. J. Pennington, R. Socher, C. D. Manning, GloVe: Global vectors for word representation in Empirical Methods in Natural Language Processing (EMNLP), (2014), pp. $1532-1543$.

31. L. R. R. Gianotti, C. Mohr, D. Pizzagalli, D. Lehmann, P. Brugger, Associative processing and paranormal belief. Psychiatry and Clinical Neurosciences 55, 595-603 (2001).

32. M. E. Webb, D. R. Little, S. J. Cropper, Once more with feeling: Normative data for the aha experience in insight and noninsight problems. Behavior Research Methods 50, 2035-2056 (2018).

33. C. Stevensonet al., Automated AUT scoring using a big data variant of the consensual assessment technique (2020).

34. J. Baer, J. C. Kaufman, Gender differences in creativity. The Journal of Creative Behavior 42, 75-105 (2008).

35. H. W. Reese, L.-J. Lee, S. H. Cohen, J. M. Puckett, Effects of intellectual variables, age, and gender on divergent thinking in adulthood. International Journal of Behavioral Development 25, 491-500 (2001). 
36. A. Abraham, Gender and creativity: An overview of psychological and neuroscientific literature. Brain Imaging and Behavior 10, 609-618 (2015).

37. A. Adnan, R. Beaty, P. Silvia, R. N. Spreng, G. R. Turner, Creative aging: Functional brain networks associated with divergent thinking in older and younger adults. Neurobiology of Aging 75, 150-158 (2019).

38. M. Palmiero, D. D. Giacomo, D. Passafiume, Divergent thinking and age-related changes. Creativity Research Journal 26, 456-460 (2014).

39. T. R. Marronet al., Chain free association, creativity, and the default mode network. Neuropsychologia 118, 40-58 (2018).

40. M. A. Runco, S. Acar, Do tests of divergent thinking have an experiential bias? Psychology of Aesthetics, Creativity, and the Arts 4, 144-148 (2010).

41. T. M. Amabile, Creativity in context: Update to the social psychology of creativity (Routledge, 2018).

42. A. Mavromatis, Hypnagogia: The Unique State of Consciousness Between Wakefulness and Sleep (Routledge \& Kegan Paul, 1987).

43. L. Prochazkovaet al., Exploring the effect of microdosing psychedelics on creativity in an open-label natural setting. Psychopharmacology 235, 3401-3413 (2018).

44. C. S. Lee, D. J. Therriault, The cognitive underpinnings of creative thought: A latent variable analysis exploring the roles of intelligence and working memory in three creative thinking processes. Intelligence 41, 306-320 (2013).

45. L. Németh, Hunspell (2020).

46. J. A. Olson, L. Suissa-Rocheleau, M. Lifshitz, A. Raz, S. P. L. Veissière, Tripping on nothing: Placebo psychedelics and contextual factors. Psychopharmacology 237, 1371-1382 (2020). 


\section{Supplementary information}

Table S1 shows descriptive statistics of the Divergent Association Task score distributions and Table S2 shows the confirmatory ANOVA results for Study 2. Figure S1 shows enjoyment across different creativity measures. Section 6.1 shows the pen-and-paper version of the Divergent Association Task that we labelled for participants as simply a "creativity task". Section 6.2 shows the algorithm code written in Python 3.

Table S1: Distribution of DAT scores across samples, after removing scores under 50.

\begin{tabular}{lrrrrr}
\hline Study & $1 \mathrm{~A}$ & $1 \mathrm{~B}$ & $1 \mathrm{C}$ & 2 & Total \\
$M$ & 78.38 & 78.15 & 80.67 & 78.28 & 78.29 \\
$S D$ & 6.35 & 5.40 & 6.20 & 6.51 & 6.48 \\
Minimum & 57.13 & 56.92 & 66.44 & 50.05 & 50.05 \\
Maximum & 93.05 & 91.61 & 91.59 & 95.74 & 95.74 \\
Skew & -0.44 & -0.38 & -0.21 & -0.57 & -0.57 \\
Kurtosis & 0.96 & 0.91 & -0.78 & 1.19 & 1.21 \\
\hline
\end{tabular}

Table S2: ANOVA table with DAT score as the dependent variable. Scores varied only slightly by demographics. Per-test $\alpha=.0167$.

\begin{tabular}{lrrrrr}
\hline Factor & $d f$ & $S S$ & $F$ & $p$ & $\eta^{2}$ \\
\hline Age & $\mathbf{1}$ & $\mathbf{1 5 5 5 . 9 8}$ & $\mathbf{3 8 . 3 9}$ & $\mathbf{< . 0 0 1}$ & $\mathbf{. 0 0 6}$ \\
Gender & $\mathbf{1}$ & $\mathbf{4 2 2 . 2 6}$ & $\mathbf{1 0 . 4 2}$ & $\mathbf{. 0 0 1}$ & $\mathbf{. 0 0 2}$ \\
Country (Australia) & 1 & 33.93 & 0.84 & .360 & .000 \\
Multilingual & 1 & 67.28 & 1.66 & .198 & .000 \\
Age $\times$ gender & 1 & 203.98 & 5.03 & .025 & .001 \\
Country $\times$ multilingual & 1 & 215.95 & 5.33 & .021 & .001 \\
Residuals & 6278 & 254423.69 & & & .990 \\
\hline
\end{tabular}




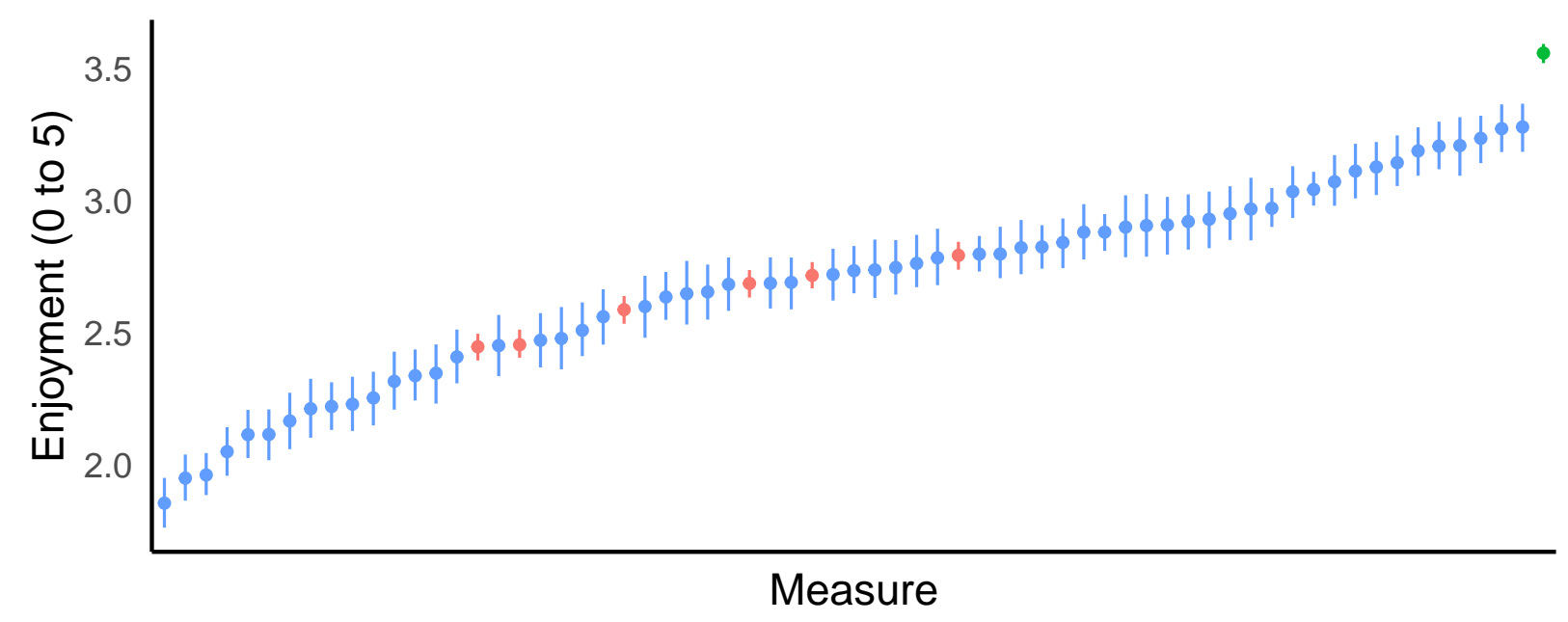

$\phi$ Alternative Uses Task $\phi$ Divergent Association Task $\quad$ Problem solving

Figure S1: Enjoyment reported after each measure in Study 2. Participants enjoyed the DAT more than any of the other measures. Dots show means and lines show bootstrapped $95 \%$ confidence intervals.

\subsection{Creativity task}

\section{Instructions}

Please write 10 words that are as different from each other as possible, in all meanings and uses of the words.

\section{Rules}

1. Only single words.

2. Only nouns (e.g., things, objects, concepts).

3. No proper nouns (i.e., no specific people or places).

4. No specialised vocabulary (e.g., no technical terms).

5. Think of the words on your own (e.g., do not just look at objects in your surroundings).

6. You will have $\mathbf{4}$ minutes to complete this task.

\section{Words}

1.

2.

3.

4.

5. 
6.

7.

8.

9.

10.

\subsection{Algorithm code}

(Code forthcoming; contact the author for a copy.) 